\title{
Preparation and Characterization of Silica and Clay-Silica Core-Shell Nanoparticles Using Sol-Gel Method
}

\author{
Olfat M. Sadek ${ }^{1}$, Safenaz M. Reda ${ }^{2^{*}}$, Reem K. Al-Bilali ${ }^{3}$ \\ ${ }^{1}$ Department of Materials Science, Institute of Graduate Studies and Research, Alexandria University, \\ Alexandria, Egypt \\ ${ }^{2}$ Chemistry Department, Faculty of Science, Benha University, Benha, Egypt \\ ${ }^{3}$ Chemistry Department, College of Science for Girls, University of Dammam, Dammam, Kingdom of Saudi Arabia \\ Email: "safenazr@yahoo.com
}

Received December 24, 2012; revised January 25, 2013; accepted February 2, 2013

Copyright (C) 2013 Olfat M. Sadek et al. This is an open access article distributed under the Creative Commons Attribution License, which permits unrestricted use, distribution, and reproduction in any medium, provided the original work is properly cited.

\begin{abstract}
Silica and montmorillonite-supported silica nanoparticles were prepared via an acid one step sol-gel process. The synthesized solids were characterized using XRD, FTIR, TEM and $\mathrm{N}_{2}$ adsorption. The effect of preparing temperatures on the structure and properties of the silica nanoparticles were studied. The results show that the increase of annealing temperature from $25^{\circ} \mathrm{C}$ to $200^{\circ} \mathrm{C}$, don't change amorphous state of silica. While for montmorillonite-supported silica the clay platelets are delaminated during the sol-gel process. TEM results showed that the average particle size of silica is increased by increasing temperature due to the particle sintering and the clay-silica nanoparticles possessed core-shell morphology with diameter of $29 \mathrm{~nm}$. The surface area measurements showed that by increasing annealing temperature the surface area was decreased due to aggregation of particle. The clay-silica sample showed lower average pore width than that of the silica prepared at $200^{\circ} \mathrm{C}$ indicating that it has a macropores structure. The adsorption efficiency of the prepared samples was tested by adsorption of protoporphyrin IX. The highest adsorption efficiency was found for $\mathrm{SiO}_{2}$ prepared at $200^{\circ} \mathrm{C}$. Temkin model describe the equilibrium of adsorption of protoporphyrin IX on caly-silica nanoparticles under different conditions.
\end{abstract}

Keywords: Silica; Clay-Silica; Sol-Gel Method; Core-Shell; Nanoparticles

\section{Introduction}

Silica nanoparticles have attracted wide interest in view of their excellent optical, electrical and thermal properties. It has been applied in many fields, such as catalysis, cosmetics, paints and drug delivery. Different approaches have been used to prepare silica nanoparticles, including the thermal methods, the sol-gel processing technique and water-in-oil (W/O) microemulsion. The sol-gel processing technique for the synthesis of silica nanoparticles has been widely used since pore volume and pore size can be controlled at the chemical solution stage [1-4]. Sol-gel-derived nano silica has many unusual properties such as an adjustable refractive index, a high porosity, a low thermal conductivity, and a low dielectric constant. Therefore, it has become increasingly attractive to use in optical, microelectronic, and thermal applications such as

\footnotetext{
${ }^{* 1,2}$ Present address: Chemistry Department, College of Science for Girls, Dammam University, Dammam, Kingdom of Saudi Arabia.
}

antiglare and antireflective coatings, thermal insulation, and other applications [5-8]. Nano structures and properties of silica are greatly dependent on experimental conditions in a sol-gel process [9-12].

Recently, considerable effort has been devoted to the fabrication of core-shell particles, which often exhibit improved physical and chemical properties of silica [4]. The core-shell can be broadly defined as comprising a core (inner material) and a shell (outer layer material). These are able to consist of a wide range of different combinations in close interaction, including inorganic/ inorganic, organic/organic, and inorganic/organic materials which the inorganic/inorganic interaction has utilized in this paper. The core-shell composite particles have been utilized in a wide range of areas, such as electronics, catalysis and separation. The inorganic samples such as clay minerals have wide applications for a long time, such as catalysts and catalytic supports [13], adsorbents of organic substances and clay based porous 
materials [13]. Since they have enhanced thermal $/ \mathrm{me}$ chanical stability, high surface area and can effectively reduce the agglomeration of particles [13].

In this paper, the silica nanoparticles have been prepared by an acid one step sol-gel method. The effect of temperatures on the structure of silica is discussed. The effect of addition of sodium montmorillonite clay (NaMMT) to properties of silica is also observed. Protoporphyrin IX (PPIX) was used as a candidate to study the adsorption efficiency of the prepared samples.

\section{Experimental}

\subsection{Chemicals}

All chemicals were used as received without further treatment. Tetraethyl-orthosilicate (acros organics), ethanol, protoporphyrin IX (sigma), montmorillonite (Saudi ceramics company). The molecular structure of protoporphyrin IX is represented in Figure 1.

\subsection{Preparation of $\mathrm{SiO}_{2}$ Nanoparticles}

$\mathrm{SiO}_{2}$ nanoparticles were prepared by the acid catalyzed through the hydrolysis of tetraethyl-orthosilicate (TEOS) using the following method: $150 \mathrm{ml}$ of TEOS was added to $155 \mathrm{ml}$ of ethanol and $190 \mathrm{ml}$ of $\mathrm{H}_{2} \mathrm{O}$ and stirred thoroughly. The mixture was vigorously stirring for 1 h. 2:3 drops of the hydrochloric acid was added to the reaction mixture as a hydrolysis catalyst to adjust the $\mathrm{pH}$ value of alkoxide reaction system. After hydrolysis, the mixture becomes a clear sol. The sols were dried in Teflon dishes at room temperature. The obtained dry xerogels were heated at temperature of $25,60,100,200^{\circ} \mathrm{C}$ in air for 25 min in order to study the effect of annealing temperature

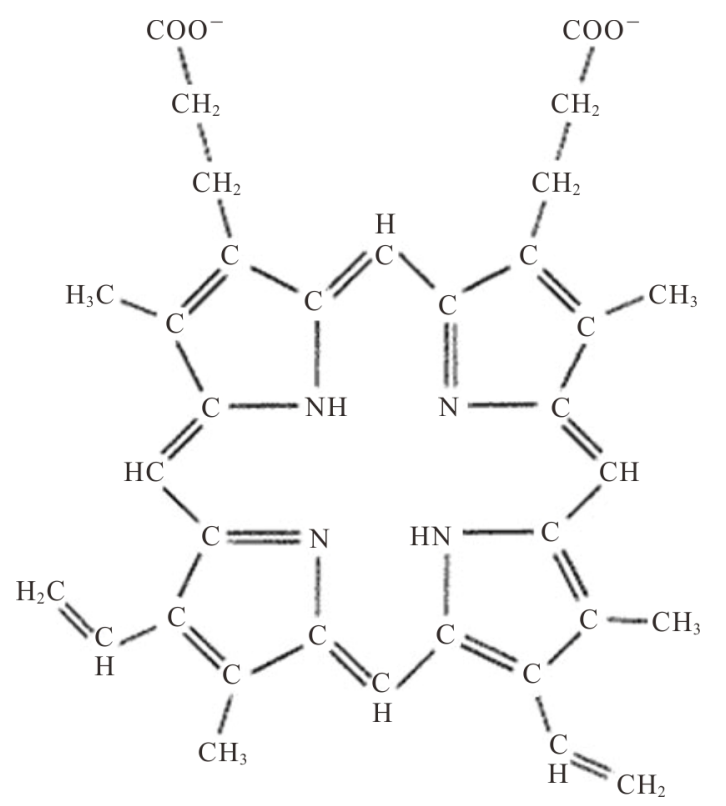

Figure 1. The structure formula of protoporphyrin IX. on structure and particle size of silica. These samples are referred to as silica-25, silica-60, silica-100 and silica200 , respectively.

\subsection{Synthesis of Montmorillonite-Silica Core-Shell}

$1 \mathrm{~g}$ of Na-MMT was dispersed in $19 \mathrm{ml}$ of distilled water to form well-dispersed slurry with magnetic stirring at room temperature for $24 \mathrm{~h}$. the mixture was then stirring ultrasonic at $25 \mathrm{kHz}$ and 100 Watt. $15 \mathrm{ml}$ TEOS and 15 $\mathrm{ml}$ ethanol were added into the clay-water slurry. The $\mathrm{pH}$ was adjusted to 3 with hydrochloric acid. The admixture was stirred vigorously at $60^{\circ} \mathrm{C}$ until a homogenous milkwhite gel was formed. The gel was aged for several days to evaporate the solvents for further condensation reaction and then dried in a vacuum at $60^{\circ} \mathrm{C}$ for $24 \mathrm{~h}$. A rigid clay-silica was obtained by these treatments was ground into powder for the analysis.

\subsection{Characterization}

X-ray powder diffraction (XRD) was recorded on Diano Corporation USA diffractometer using Co-radiation $(\lambda=$ $0.179 \mathrm{~nm}$ ), to study the crystal structure and crystallinity of the $\mathrm{SiO}_{2}$ nanoparticles. The surface properties namely BET surface area and mean pore radius (r) were determined from $\mathrm{N}_{2}$ adsorption isotherm using conventional or classical volumetric apparatus. Surface Morphology and microstructure was investigated by TEM (Jeol JXA840 electron probe microanalyzer). The UV-Vis absorption was obtained with a (UVD-2960) labomed spectrophotometer. FT-IR was recorded using Jasco FT/IR spectrometer in wavenumber range from $4000-400 \mathrm{~cm}^{-1}$.

\subsection{Adsorption of Protoporphyrin IX Molecule on Silica and Clay-Silica Core-Shell}

Adsorption of PPIX with the synthesized $\mathrm{SiO}_{2}$ nanoparticles were carried out as batch tests in $250 \mathrm{ml}$ flasks. 25 $\mathrm{ml}$ of PPIX solution with desired concentration is prepared in ethanol as a solvent. The $\mathrm{pH}$ of the solution was adjusted using $0.1 \mathrm{~N} \mathrm{HCl}$ or $\mathrm{NaOH}$ solutions. A $0.025 \mathrm{~g}$ of nano- $\mathrm{SiO}_{2}$ powder was then added to the solution, the final silica concentration is $1 \mathrm{~g} / \mathrm{l}$. The obtained suspension was immediately shaken for $1 \mathrm{~h}$. After the mixing time elapsed, the suspension was allowed to settle and the supernatant was analyzed using UV-Vis spectrophotometer for the residual dye concentration. The concentration of the remaining dye in the solution was determined by measuring the absorbance intensity at $485 \mathrm{~nm}$ [14]. All experiments were performed at room temperature. The investigated ranges of the experimental variables were as follows: dye concentration $\left(10^{-4}, 5 \times 10^{-5}\right.$ and $\left.1.5 \times 10^{-5} \mathrm{~mol} / \mathrm{l}\right)$, $\mathrm{pH}$ of solution $(2,10)$, dosage $(1$ $\mathrm{g} / \mathrm{l})$ and mixing time (1 -8 days). 


\section{Results and Discussion}

\subsection{Characterization}

XRD patterns of the $\mathrm{SiO}_{2}$, Na-MMT and montmorillonitesilica nanocomposite are shown in Figure 2. The results show that there is any characteristic peaks of $\mathrm{SiO}_{2}$ and the appeared peaks are amorphous in nature. This indicates that the annealing temperature had no significant influence on the phase formation of the $\mathrm{SiO}_{2}$ nanoparticles till $200^{\circ} \mathrm{C}$. The results are in good agreement with that reported in lecture [15]. Figure 2(e) shows XRD patterns of the montmorillonite-silica core-shell. The figure shows the broad peak at $22^{\circ}$ corresponds to the typical diffraction of amorphous $\mathrm{SiO}_{2}$ (Si-O short-order structure) [16]. The (001) diffraction peaks of Na-MMT at $6^{\circ}$ are vanished, Figure 2(f), suggesting that clay platelets are delaminated during the sol-gel process.

FTIR spectra have been used to characterize the prepared samples, Figure 3. As shown in this Figure the two broad bands at 3470 and $1639 \mathrm{~cm}^{-1}$ are ascribed to the stretching modes and $\mathrm{H}-\mathrm{O}-\mathrm{H}$ bending vibration of the free or absorbed water. Strong absorptions at 1077, 798,
$474 \mathrm{~cm}^{-1}$ indicate the formation of silica network. The absorption band at $1077 \mathrm{~cm}^{-1}$ attributed to Si-O-Si of the $\mathrm{SiO}_{4}$ tetrahedron and the absorption band at $474 \mathrm{~cm}^{-1}$ associated with $\mathrm{Si}-\mathrm{O}-\mathrm{Si}$ or $\mathrm{O}-\mathrm{Si}-\mathrm{O}$ bending mode. These observations afford proof of the generating silica nanoparticles [12].

Figure 3(e) illustrates FT-IR spectra of clay -modified silica. After modification with clay, the bands at about $3470 \mathrm{~cm}^{-1}$ corresponds to the stretching vibration of surface hydroxyl groups, and the bands at 956 and $798 \mathrm{~cm}^{-1}$ attribute to the $-\mathrm{OH}$ bending vibration. The intensity of these characteristic bands of the surface hydroxyl groups increase indicating that the condensation reactions takes place not only between the silica sol particles themselves but also between the sol particles and hydroxyl groups on clay surfaces. A new band at about $545 \mathrm{~cm}^{-1}$ appears which is assigned to Al-O-Si of clay. The FT-IR spectra (Figure 3(e)) shows no obvious changes of the $-\mathrm{OH}$ bands at about 3627,916 and $847 \mathrm{~cm}^{-1}$. These results suggest that surface hydroxyl groups of the clay were reacted with the hydrolyzed TEOS, and the resultant silica is covalently attached on the clay surfaces [13].

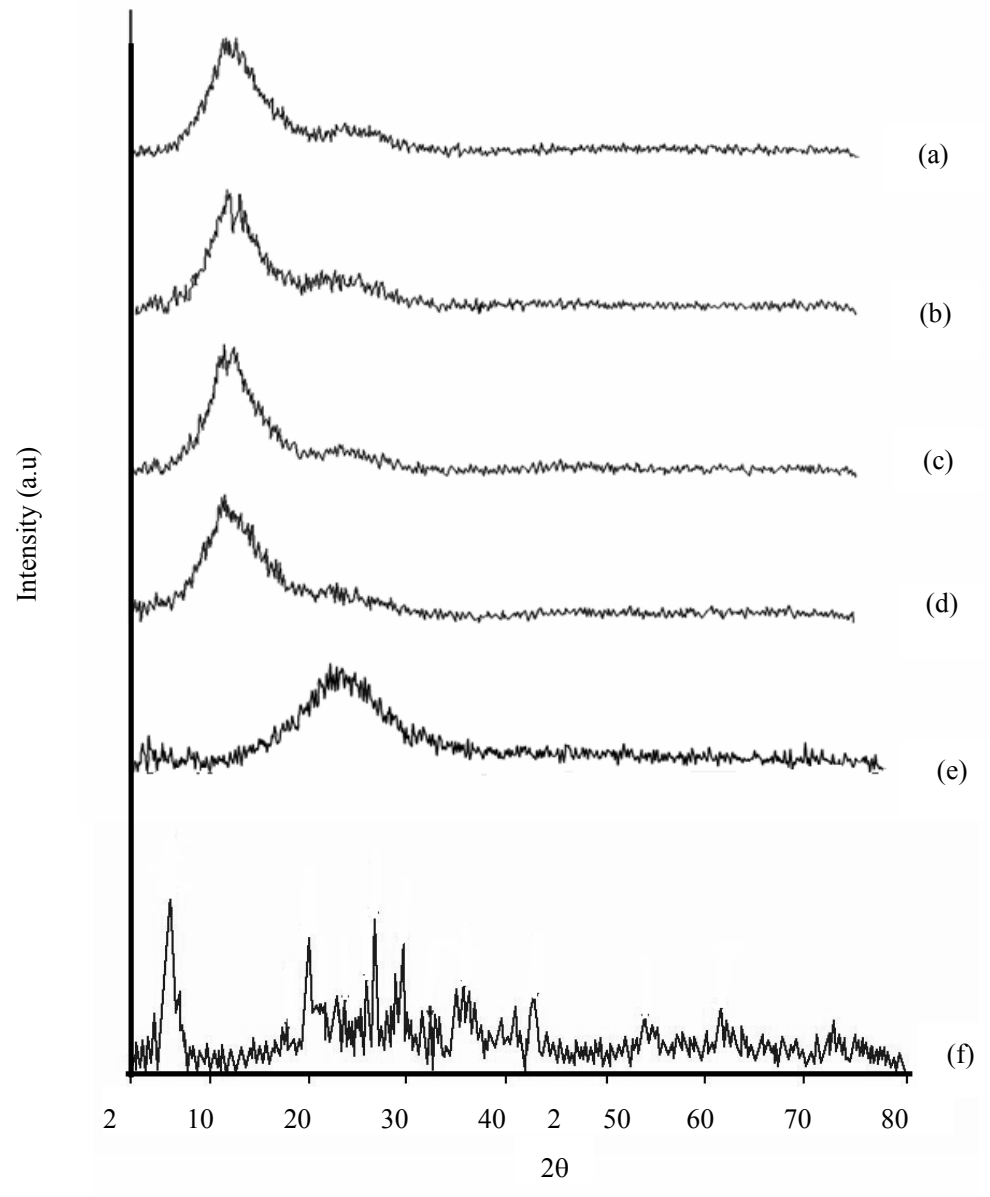

Figure 2. X-ray diffraction patterns for silica-25 (a), silica-60 (b), silica-100 (c), silica-200 (d), clay-silica nanocomposite (e), and Na-MMT (f). 


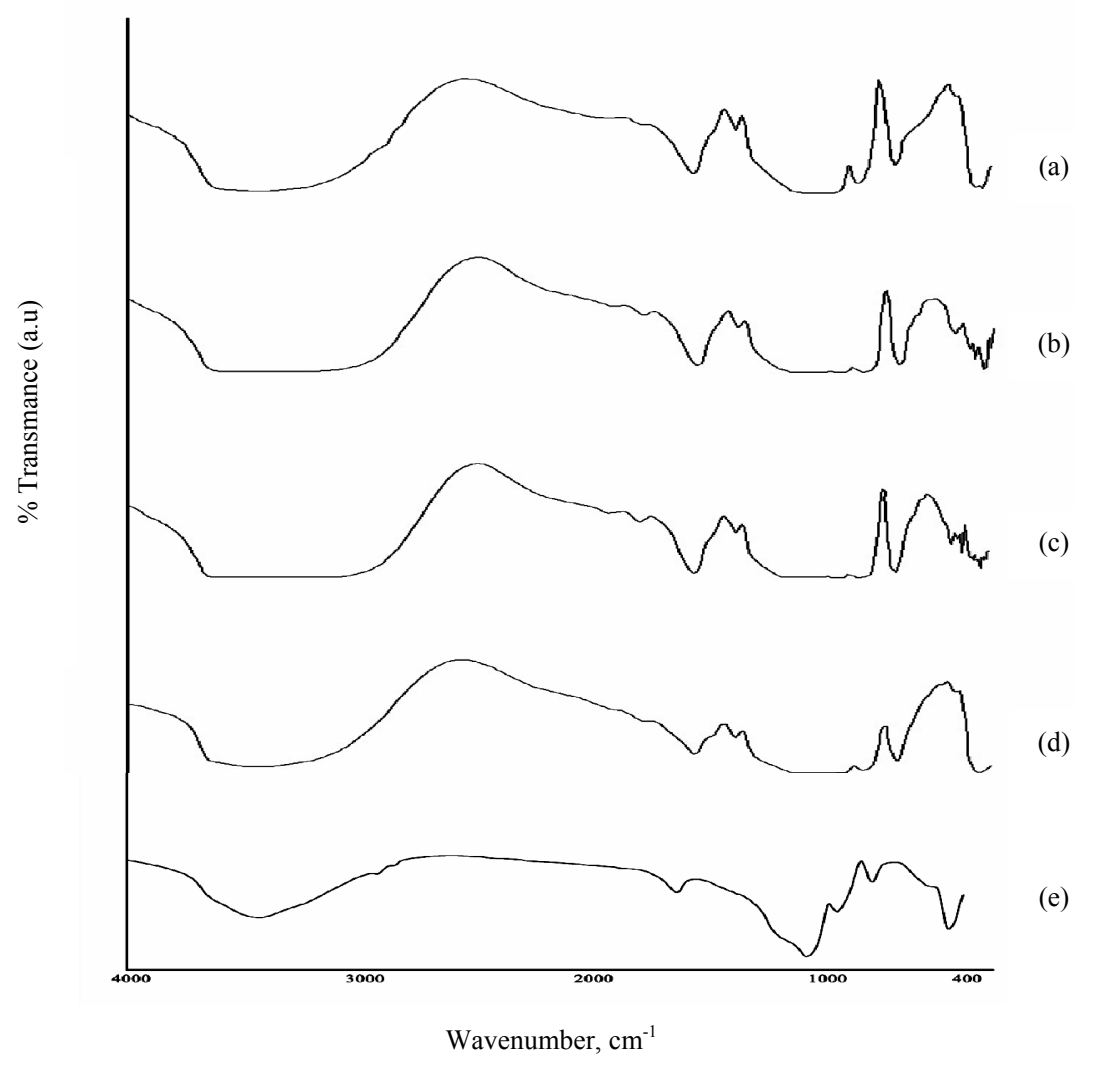

Figure 3. FTIR specrta of silica-25 (a), silica-60 (b), silica-100 (c), silica-200 (d) and clay-silica nanocomposite (e).

Figure 4 shows TEM images of pure silica and silicaclay samples. The compacted spherical morphology is clearly visible in the TEM images for all samples. Figures 4(a)-(d) illustrate the TEM photographs of silica nanoparticles annealed at different temperatures. The average particle size for silica-25, silica-60, silica-100 and silica-200 was $27,30,65$, and $202 \mathrm{~nm}$, respectively. As we seen, the average particle size increase by increasing temperatures. The main reason is that the growth of particles by increasing temperature. It is evident that the higher annealing temperature the narrower size distribution was observed. This may be due to the lowered viscosity at higher temperatures, which increase the particle aggregation during precipitation process. The transmission electronic micrographs (TEM) of the obtained claysilica nanocomposites are shown in Figure 4(e). From the insets of this figure, it is evident that the final composite nanoparticles possessed core-shell morphology with diameter of $29 \mathrm{~nm}$ was obtained. This is easily understood because more silica network was formed on the clay and surrounding the clay plates [13].

\subsection{Nitrogen Adsorption-Desorption Measurements}

The nitrogen adsorption-desorption isotherms are carried out to investigate the surface morphology and physical properties of the silica samples annealed at different temperatures, Figure 5. A comparison between the hysteresis loops of these samples indicates that they are of type H4 except for silica-200 for which a superposition of H3 loops is observed. The isotherm of silica-200 (Figure 5(d)) presents a type-H3 hysteresis loop ascribed to aggregates (loose assemblages) of plate-like particles forming slit-shaped pores [15]. All of the isotherms are closet in shape to type I because hysteresis occurs during the desorption branches, according to the BDDT classification [15]. However, there are noticeable differences in the shape of their hysteresis loops. However, silica-200 showed shape to type II.

Figure 5(e) shows the adsorption-desorption isotherm for clay-silica core shell. This Figure shows that the clay modified silica present a type-H3 hysteresis loop, which can be attributed to mesoporous structure containing open slit-shaped capillaries with wide bodies and narrow short necks [13].

The BET surface areas $\left(\mathrm{S}_{\mathrm{BET}}\right)$, total pore volumes $\left(\mathrm{V}_{\mathrm{p}}\right)$ and average pore radius (r) for all examined samples are shown in (Table 1). It shows that pristine silica-25 has an obvious increase in surface area compared with the silica prepared at different temperature [15]. The BET surface 


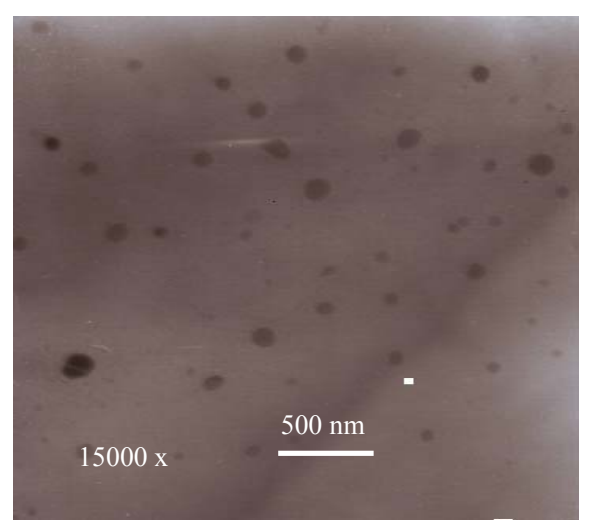

(a)

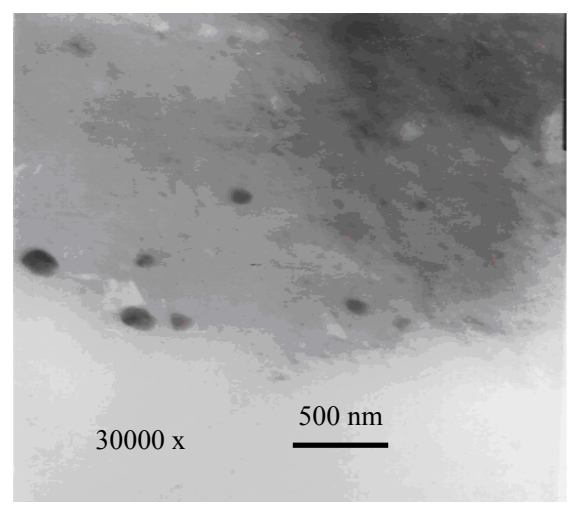

(c)

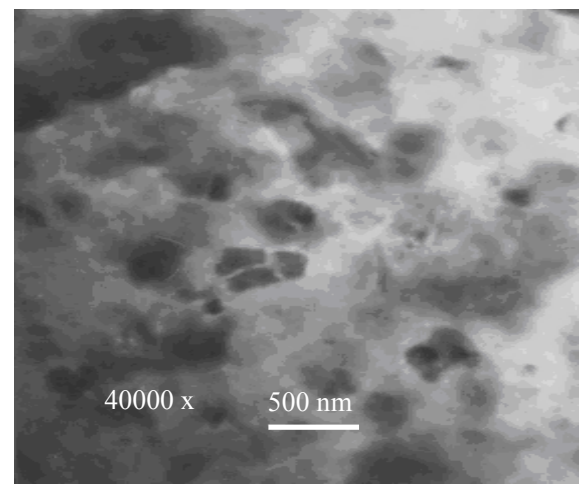

(e)

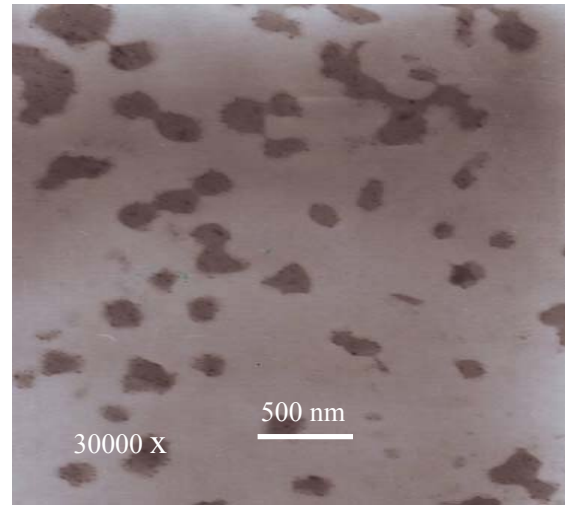

(b)

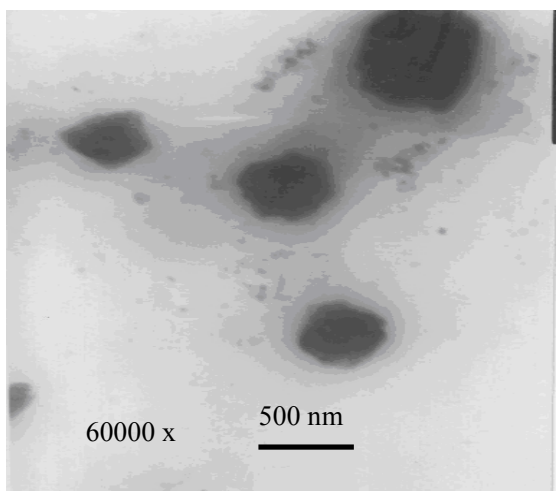

(d)

Figure 4. TEM images for silica-25 (a), silica-60 (b), silica-100 (c), silica-200 (d) and clay-silica nanocomposite (e).

areas are decreased by increasing annealing temperature for silica samples due to increase aggregation of particle by increasing annealing temperature. The clay silica sample shows higher average pore width than that of the pure silica-200 (as shown in Table 2), indicating again macropores and interstices are formed between the silica particles, while mesoporous silica networks are formed in the latter case. Under the acid-catalyzed conditions the sol-gel process involves complete hydrolysis reaction, replacing alkoxide groups $\left(-\mathrm{OC}_{2} \mathrm{H}_{5}\right)$ with hydroxyl groups $(-\mathrm{OH})$, and subsequent condensation reactions between silanol groups $(\mathrm{Si}-\mathrm{OH})$ and hydroxyl groups located on the clay surfaces to form covalent bonds [15]. Meanwhile, the hydrolyzed TEOS also self condense to form large continuous silica networks with mesoporous structure, especially in the case of the high TEOS/clay. As a result, clay platelets are covalently attached with silica, and the growth of the mesoporous networks penetrated into clay interlayer leads to the formation of the exfoliation structure of clay platelets. Measurements of 


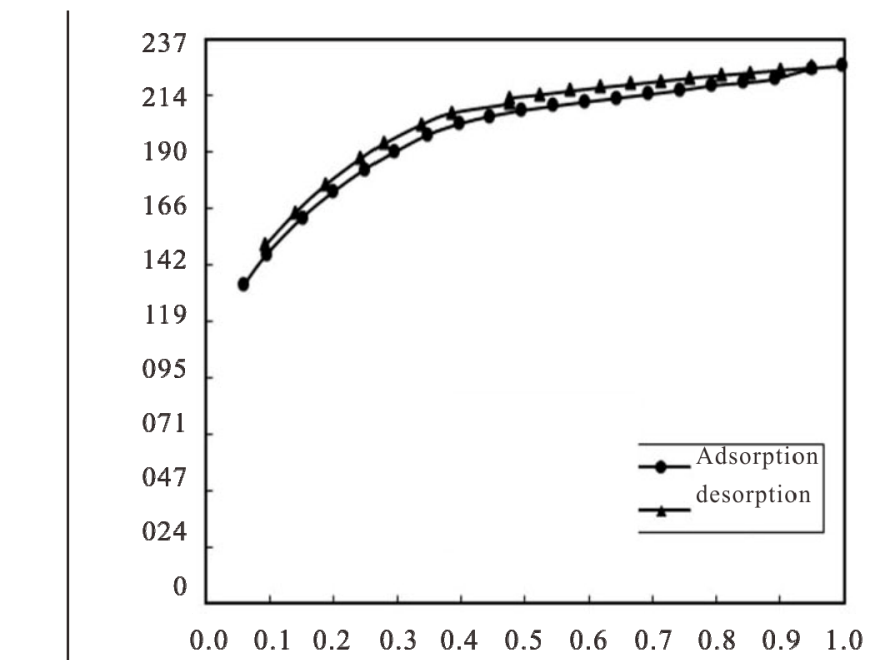

(a)

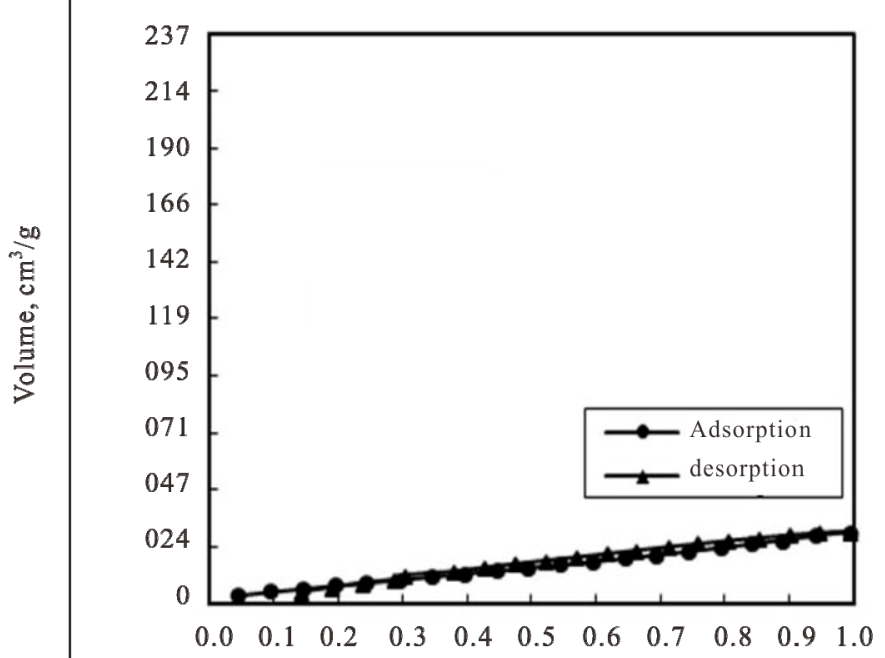

(c)

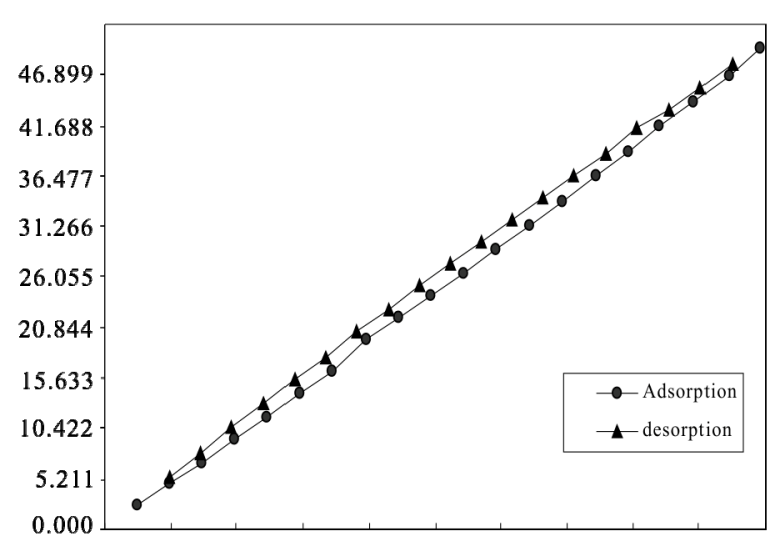

$0.0000 \quad 0.1000 \quad 0.20000 .30000 .40000 .50000 .60000 .70000 .80000 .90001 .0000$

(e)

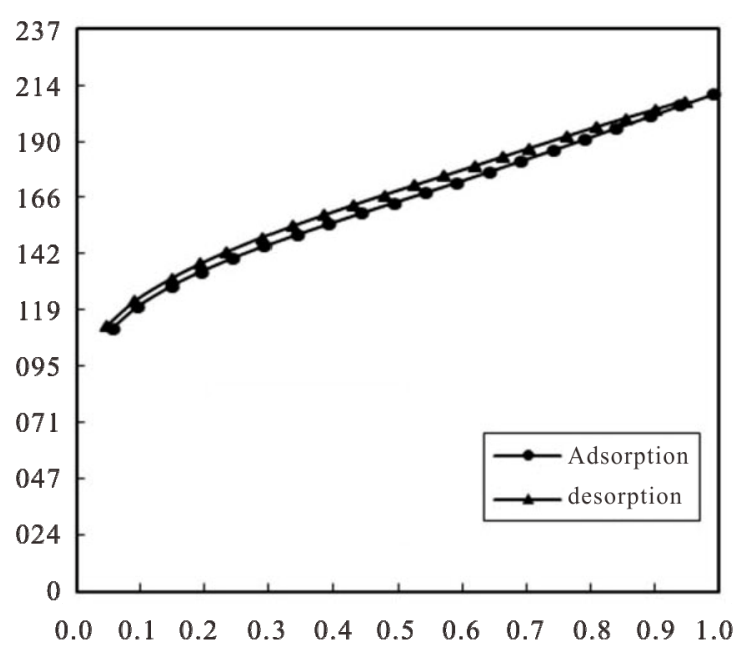

(b)

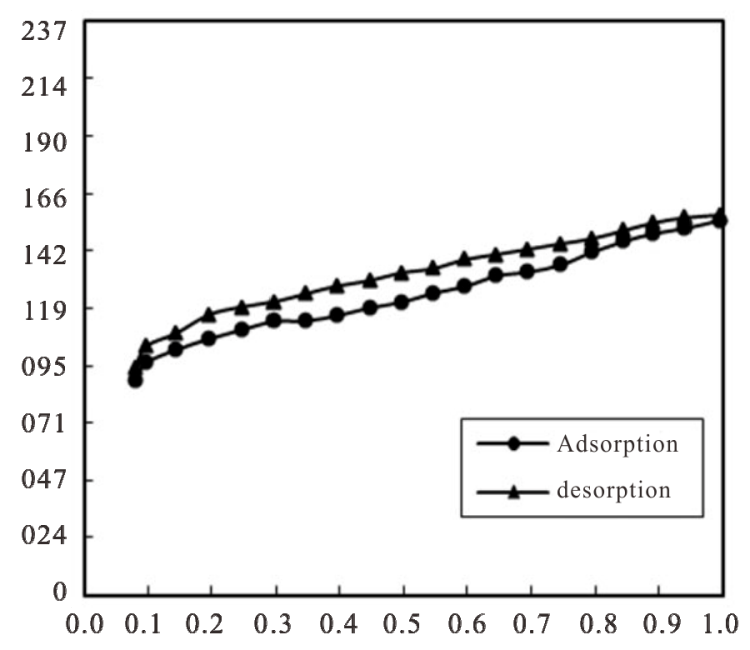

(d)

Relative pressure, $\mathrm{P} / \mathrm{P}_{0}$

Figure 5. Nitrogen adsorption-desorption isotherm for silica-25 (a), silica-60 (b), silica-100 (c), silica-200 (d) and clay-silica (e). 
Table 1. Parameters resulting from the analysis of $\mathrm{N}_{2}$ adsorption isotherms of the silica samples.

\begin{tabular}{cccc}
\hline Samples & BET surface area $\left(\mathbf{S}_{\mathbf{B E T}}, \mathbf{~ m}^{\mathbf{2}} \mathbf{g}\right)$ & Total pore volume $\left(\mathbf{V}_{\mathbf{b}}, \mathbf{c m}^{\mathbf{3}} \mathbf{g}^{-\mathbf{1}}\right)$ & Average pore width $\left(\mathbf{r}, \mathbf{A}^{\circ}\right)$ \\
\hline Silica-25 & 596.61 & 0.3498 & 1.47 \\
Silica-60 & 451.04 & 0.3247 & 1.80 \\
Silica-100 & 338.32 & 0.2476 & 1.83 \\
Silica-200 & 338.32 & 0.0466 & 1.83 \\
Clay-silica core-shell & 79.59 & 0.0763 & 2.40 \\
\hline
\end{tabular}

Table 2. Parameters resulting from the $t$-analysis for all investigated samples.

\begin{tabular}{lcccccc}
\hline Samples & $\begin{array}{c}\text { Pore surface area } \\
\left(\mathbf{S}_{\mathbf{t}}, \mathbf{~ m}^{2} \mathbf{g}\right)\end{array}$ & $\left(\mathbf{S}_{\text {ext }}, \mathbf{m}^{2} / \mathbf{g}\right)$ & $\begin{array}{c}\text { Microporous } \\
\text { surface area } \\
\left(\mathbf{S}_{\text {micro }}, \mathbf{m}^{2} / \mathbf{g}\right)\end{array}$ & $\begin{array}{c}\text { Mesoporous } \\
\text { surface area } \\
\left(\mathbf{S}_{\text {meso }}, \mathbf{m}^{\mathbf{2}} / \mathbf{g}\right)\end{array}$ & $\begin{array}{c}\text { Microporous volume } \\
\left(\mathbf{V}_{\text {micro }}, \mathbf{c m}^{\mathbf{3}} \mathbf{g}^{-1}\right)\end{array}$ & $\begin{array}{c}\text { Mesoporous volume } \\
\left(\mathbf{V}_{\text {meso }}, \mathbf{c m}^{\mathbf{3}} \mathbf{g}^{-1}\right)\end{array}$ \\
\hline Silica-25 & 547.51 & 70.32 & 526.3 & 21.21 & 0.256 & 0.0937 \\
Silica-60 & 354.35 & 102.6 & 348.4 & 5.95 & 0.177 & 0.1477 \\
Silica-100 & 145.5 & 61.73 & 276.6 & 1.50 & 0.1503 & 0.0973 \\
Silica-200 & 36.66 & 28.85 & 9.622 & 27.04 & 0.0042 & 0.04240 \\
$\begin{array}{l}\text { Clay-silica } \\
\text { core-shell }\end{array}$ & 66.53 & 50.97 & 28.62 & 37.91 & 0.0014 & 0.0749 \\
\hline
\end{tabular}

the pore size showed a decreased in pore size by increasing annealing temperature for pure silica reaching the mesoporous region. While for the clay-silica sample has a pore size around $2 \mathrm{~nm}$, the clay-silica sample has a larger amount of pores in the micropore region than in the mesopore region.

\subsection{Analysis by $t$-Plot}

The statistical thickness, $t$, of the physically adsorbed layer as a function of relative pressure is estimated based on the de Boer equation given in equation [15]:

$$
t=\left(13.9 /\left(0.034-\log \frac{P}{P^{o}}\right)\right)^{1 / 2}
$$

The volume of gas adsorbed versus $t$ known as $t$-plot is then used for the pore analysis. Figure 6 shows two distinct linear stages in the $t$-plots, initial stage of adsorption on the pore wall; intermediate stage is extrapolated to the adsorption volume ordinate, the positive intercept at the ordinate measures the micropore volume of the sample that have been filled by adsorbed molecules at the beginning of the intermediate stage. Similarly, the linear portion of the last stage measures the external surface area and the total pore volume exclusive of external surface pore volume [15]. Therefore $t$-plot analysis is more appropriately used for the samples containing both micropores and mesopores [15]. The $t$-plot pore surface areas of the silica samples and caly-silica, $S_{t}$, calculated from the slopes of the initial linear stage of $t$-plots are similar to the corresponding BET surface areas except that for silica annealed at 100 and $200^{\circ} \mathrm{C}$ (Table 2). The micropore pore volumes, $V_{\text {PORE,MICROPOROUS }}$ is large the corresponding

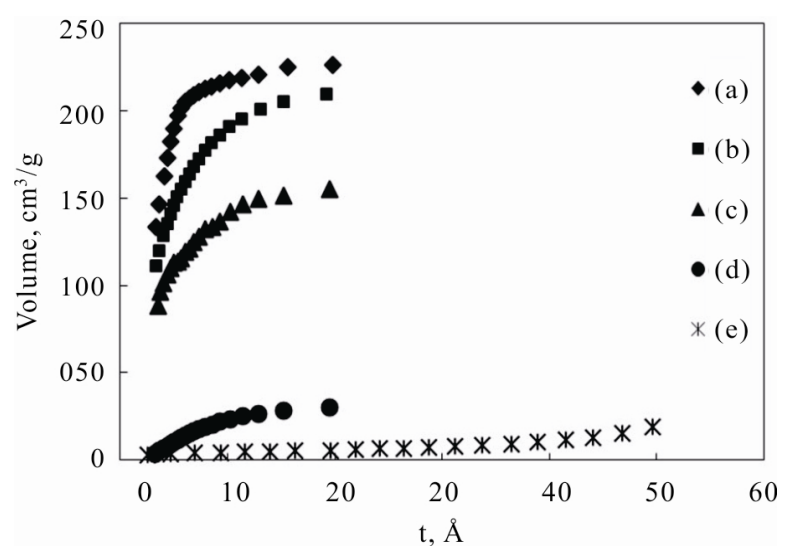

Figure 6. $t$-analysis for silica-25 (a), silica-60 (b), silica-100 (c), silica-200 (d) and clay-silica (e).

mesopore pore volumes, $V_{\text {PORE,MESOPOROUS }}$ (Table 2) except silica annealed at 200 and clay-silica samples. This is not surprising as the pore filling of micropores is due to gaseous adsorption that occupies larger surface area than the pore filling of mesopores with liquid-like capillary condensation. Table 2 shows also that, for all samples external surface $S_{\text {ext }}$ areas are less than the corresponding BET surface areas. It can be noted that, if $S_{\mathrm{t}}$ (calculated from the initial slope) is considered as the total surface area arising from all pores, the microporous surface area $S_{\text {mic }}$ will be $\left\{S_{\mathrm{t}}-\left(S_{\mathrm{t}, \text { MESOPOROUS }}+\right.\right.$ $\left.\left.S_{\mathrm{t} \text {,EXTERNAL }}\right)\right\}$ that is $526,348,276,9.6$ and $28.6 \mathrm{~m}^{2} / \mathrm{g}$ for silica prepared at $25,60,100,200$ and clay-silica, respectively. The total pore volume of the silica at $25^{\circ} \mathrm{C}$ is found to be maximum, $0.349 \mathrm{~cm}^{3} / \mathrm{g}$; and is minimum in case of the silica at $200^{\circ} \mathrm{C}, 0.0466 \mathrm{~cm}^{3} / \mathrm{g}$. Also, the microspore volume of the silica at $25^{\circ} \mathrm{C}$ is found to be the 
largest, $0.256 \mathrm{~cm}^{3} / \mathrm{g}$. This agrees well with the differences in corresponding $S_{t}$ surface areas.

\subsection{Adsorption of Protoporphyrin IX Molecule on Nano Particles of Silica and Clay-Silica}

The adsorption process was carried out using silica nano practices pretreated at $25^{\circ}, 60^{\circ}$, and $200^{\circ} \mathrm{C}$, as well as clay-silica nano particles. It was observed that silica- 25 has not ability to absorb the PPIX, there is no change in PPIX concentration in the medium till two days. Other samples: silica-60, silica-100 and silica-200 show slight capability to interact with PPIX. The efficiency of the adsorption process ( $\%$ eff.) can be determined using the following equation:

$$
\% \text { eff }=\frac{c_{\mathrm{o}}-c_{e q}}{c_{\mathrm{o}}} \times 100
$$

where $c_{\circ}$ and $c_{e q}$ are the concentration of PPIX at initial and equilibrium time respectively, $(\mathrm{mg} / \mathrm{l})$.

Figure 7 shows the adsorption efficiency of protoporphyrin IX on different $\mathrm{SiO}_{2}$ surfaces after two days, as function of annealing temperature. Obviously, the silica200 exhibited the highest adsorption efficiency with $37 \%$ in 2 days, while silica- 100 showed $34 \%$ and silica- 60 showed $25 \%$ adsorption efficiency in the same test period. The silica-25 don't show any change in adsorption in the same time. Also, it was observed that silica-200 show slight increase in its efficiency after the third day to attain $52 \%$, while is no improvement of the efficiency of silica-60 and silica 100 . The highest adsorption efficiency of silica-200 in may be attributed to its large pore radius $(7.6 \mathrm{~nm})$ as observed in BET measurements, indicating that the pore of meso-type considers to the suitable void type permit the interaction of PPIX molecules with silica surfaces. Also, the surface area of mesopores increased dramatically when the annealing temperature increase to $200^{\circ} \mathrm{C}$.

Figure 8 shows the UV-Vis absorption spectra of the PPIX in supernatant solution before and after the adsorption on clay-silica nano particles at $\mathrm{pH}=10$. After adsorption, the intensity of the sort band at $402 \pm 0.5 \mathrm{~nm}$ was decreased as expected due to the reduction in the PPIX concentration in solution due to their interaction with clay-silica nano particles.

The initial dye concentration is another important variable that can affect the adsorption process. The effect of initial concentration of protoporphyrin IX at $\mathrm{pH}=10$ were $\left(10^{-4}, 5 \times 10^{-5}\right.$ and $\left.1.5 \times 10^{-5} \mathrm{~mol} / \mathrm{l}\right)$ was studied on their adsorption onto clay-silica powder under previously determined optimum conditions. The results, in terms of efficiency versus initial concentration of dye, are indicated in Figure 9. According to Figure 9, efficiency of PPIX increases by increasing dye concentration and doesn't change by increasing concentration up to $1.5 \times$ $10^{-5} \mathrm{M}$. This may be attributed to the saturation of adsorption sites on clay-silica by dyes at this concentration. It was noted that the incorporation of clay with silica lead to increase in the mesopores surface area leading to maximize the interaction between PPIX and the claysilica nano particles in point of view their rate and efficiency. We notes that the values of adsorption efficiency of the clay-silica nano particles at $\mathrm{pH}=10$ is better than the pure silica and clay and reaches to $87 \%$ at initial concentration $\left(1 \times 10^{-5} \mathrm{~mol} / \mathrm{l}\right)$ of PPIX. This can be explained on the bases of the dissolution of silica network surrounding the clay layers at $\mathrm{pH}=10$ and the clay plates become available for adsorption of PPIX [15], while in neutral and acidic medium $(\mathrm{pH}=2)$ less clay surface is available to adsorb PPIX, as shown by the proposed structure model (Figure 10).

In order to investigate the adsorption equilibrium of the adsorption process, the concentration of PPIX was determined. The adsorbed amount of PPIX $\left(q_{t}\right)$ in $\mathrm{mg} / \mathrm{g}$

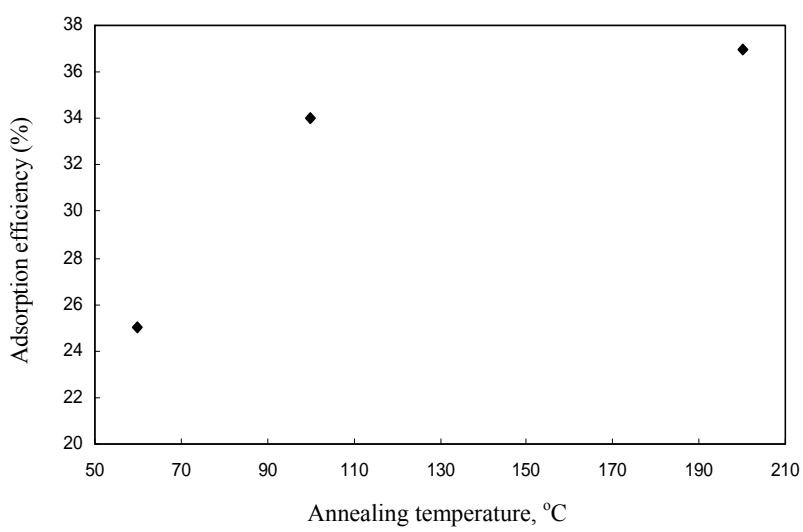

Figure 7. Dependence of adsorption efficiency of protoporphyrin IX on the annealing temperature of $\mathrm{SiO}_{2}$ samples after 2 days. $\left(\mathrm{SiO}_{2}\right.$ dosage $=1 \mathrm{~g} / \mathrm{l}$, initial $\mathrm{pH} 7$, initial dye concentration $\left.=1.5 \times 10^{-5} \mathrm{~mol} / \mathrm{l}\right)$.

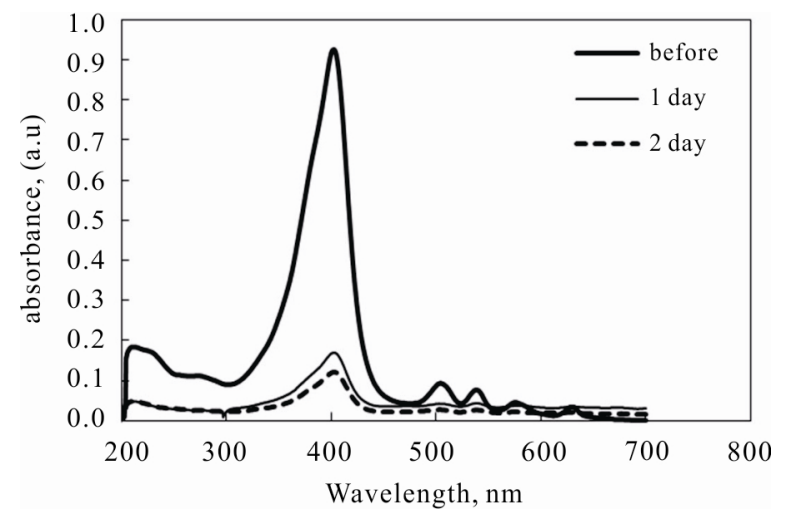

Figure 8. UV-Vis spectra for PPIX before and after adsorption on clay-silica (initial concentration of PPIX $=1 \times 10^{-5}$ $\mathrm{M}, \mathbf{p H}=\mathbf{1 0})$. 


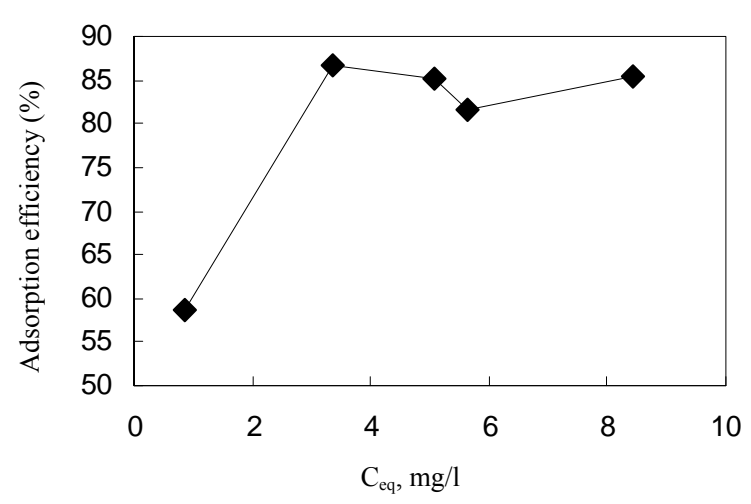

Figure 9. Effect of concentration on adsorption efficiency of PPIX on clay-silica samples. (clay-silica dosage $=2 \mathrm{~g} / \mathrm{l}$ and pH $=10)$.

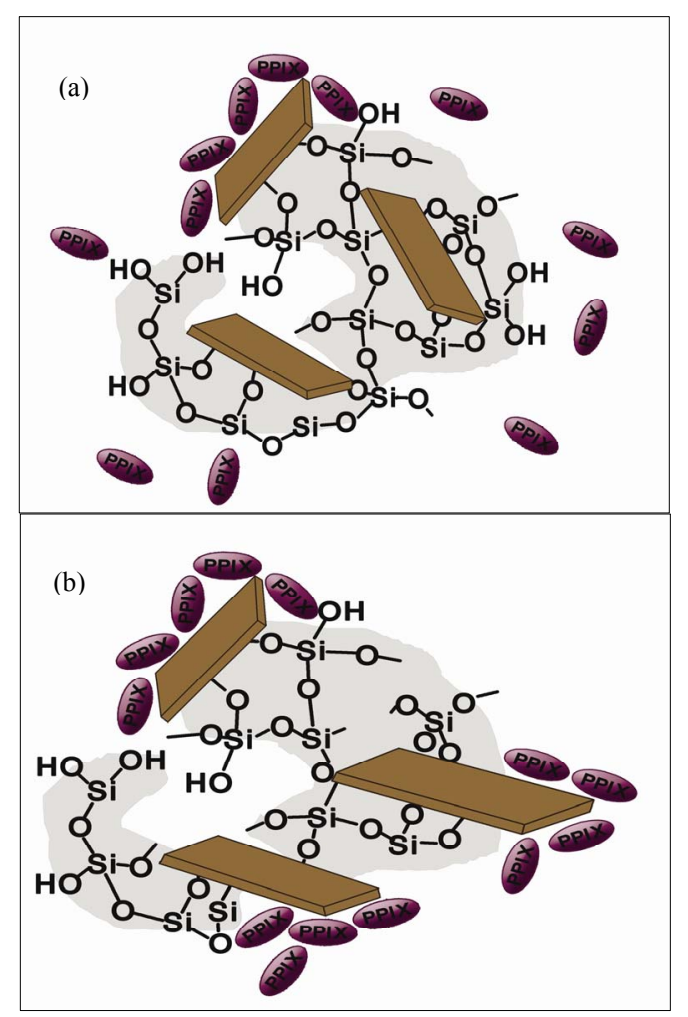

Figure 10. Schematic representation of proposed structure models of clay-silica nanocomposite after adsorption of PPIX $\left(C_{o}=10 \mu \mathrm{M}\right)$ at (a) neutral medium and $\mathrm{pH}=2$, and (b) $\mathrm{pH}=10$.

dry sample, on the silica surfaces at any time $(t)$ in min., can the calculated from the equation:

$$
q_{t}=\frac{\left(c_{o}-c_{t}\right)}{m} v
$$

where $c_{t}$ is the PPIX concentration in the supernatant at any time $(\mathrm{mg} / \mathrm{l}) v$ is the solution volume (l), and $m$ is the weight of the dray sample (g).
Figure 11 shows the adsorption isotherm of PPIX onto clay-silica nano particles at $\mathrm{pH}=10$ which was determined by plotting the amount PPIX adsorbed by the nano particles $\left(q_{e q}\right)$ in $\mathrm{mg} / \mathrm{g}$ dray sample versus the equilibrium concentration of PPIX $\left(c_{e q}\right)$ in $\mathrm{mg} / \mathrm{l}$ in solution. The shape of this isotherm is corresponding to s-type, subgroup-3 according to Giles classification [13]. The scurve indicates that there is a solute-solute attractive force of the surface. Also, it indicates that the sorption of PPIX may be inhabited by a competing reaction with the solution, such as a complexation reaction with the surfaces. This means that the PPIX have low affinity to thesurface, but as soon as surface is covered by PPIX molecules, other molecules are adsorbed more easily. There are a second layer can formed readily, thus, it follows up the sub-group 3.

It is very important to establish the most appropriate correlation for the equilibrium data and optimize the adsorption system design to adsorb PPIX on clay-silica nano particles. Four common adsorption equilibrium models (Langmuir, Frundlish, Tembkin and DubininRadushkevish) were tested. The adsorption data were analyzed according to the linear from the previous models, the figures not included, and the correlation coefficient, $\mathrm{R}^{2}$ was determine which is follow the order:

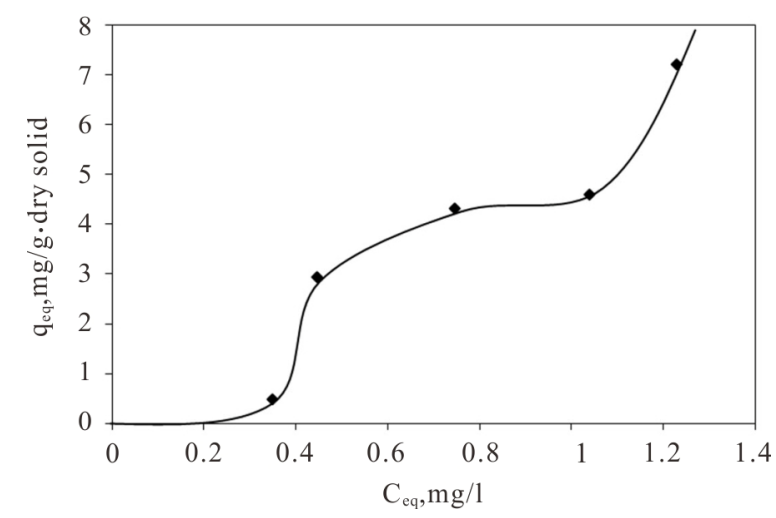

Figure 11. Adsorption isotherm of protoporphrin IX on clay -silica sample. (clay-silica dosage $=1 \mathrm{~g} / \mathrm{l}$, initial $\mathrm{pH}=10$ ).

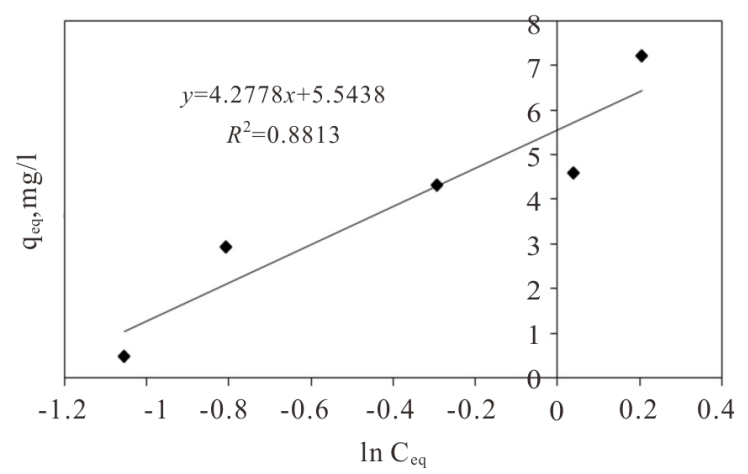

Figure 12. Temkin adsorption model for adsorption of protoporphyrin IX on clay-silica sample at $\mathbf{p H}=10$. 
Temkin $(0.88)>$ Dubinin-Radushkevich $(0.76)>$ Freundlich $(0.75)>$ Langmuir $(0.32)$. According to $\mathrm{R}^{2}$ values, the most probable models can describe the interaction of PPIX on clay-silica nano particles is Temkin isotherm. It has generally been applied the following form [17].

$$
q_{e q}=\beta \ln \left(A c_{e q}\right)
$$

Their linear form can be expressed as:

$$
q_{e q}=\beta \ln A+\beta \ln c_{e q}
$$

where $\beta$ is a constant, refers to the differential surface capacity per unit binding energy, $(\beta=R T / b)$, $\mathrm{T}$ is absolute temperature, $\mathrm{R}$ is the gas constant. $\beta$ is inversely proportional to the heterogeneity and extent of adsorbate-adsobate interaction. The adsorption data can he analyzed according to Equation 5. Therefore, a plot of $q_{e q}$ versus $\operatorname{lnc}_{e q}$ as shown in Figure 12, enable to determine the constants $A$ and $\beta$. From the slope of the straight line obtained the value of $\beta$ can he obtained, $\beta=4.277$, then the value of $A$ can he calculated from the intercept of the line with the coordinate, $A=3.654$.

\section{Conclusion}

Silica and clay-silica core-shell nanoparticle were fabricated by one-step catalyzed sol-gel method. The effect of temperature on properties of resulting silica was studied. The prepared samples were characterized using XRD, FT-IR, TEM and $\mathrm{N}_{2}$ adsorption. The obtained results showed that increasing temperature from 25 to $200^{\circ} \mathrm{C}$ increase particle size and decease BET surface area due to the particle aggregation. As confirmed from TEM the clay-silica nanoparticles possessed core-shell morphology with diameter of $29 \mathrm{~nm}$. The pore structure properties such as external surface, microporous surface area, mesoporous surface area, total pore volume, microspore volume, mesopore volume are evaluated using $t$-analysis method. It was found that the microporous surface area is $526,348,276,9.6$ and $28.6 \mathrm{~m}^{2} / \mathrm{g}$ for silica prepared at 25 , $60,100,200^{\circ} \mathrm{C}$ and clay-silica, respectively. The adsorption efficiency of the prepared samples for porotoporphrin IX showed that $\mathrm{SiO}_{2}$ prepared at $200^{\circ} \mathrm{C}$ has the highest adsorption efficiency due to its large pore radius $(7.6 \mathrm{~nm})$. The results of adsorption showed also that the adsorption of protoporphyrin IX on clay-silica nanoparticles core-shell is better described by the Temkin model.

\section{Acknowledgements}

The authors very gratefully thank the King Abdulaziz City for Science and Technology for cooperation and support (AT-17-73).

\section{REFERENCES}

[1] R. Vacassy, R. J. Flatt, H. Hofmann, K. S. Choi and R. K. Singh, "Synthesis of Microporous Silica Spheres," Journal of Colloid and Interface Science, Vol. 227, No. 2, 2000, pp. 302-315. doi:10.1006/jcis.2000.6860

[2] F. Iskandar and O. K. Mikrajuddin, "In Situ Production of Spherical Silica Particles Containing Self-Organized Mesopores," Nano Letters, Vol. 1, No. 5, 2001, pp. 231-234. doi:10.1021/n10155227

[3] G. De, B. Karmakar and D. Ganguli, "Hydrolysis-Condensation Reactions of TEOS in the Presence of Acetic Acid Leading to the Generation of Glass-Likesilica Microspheres in Solution at Room Temperature," Journal of Materials Chemistry, Vol. 10, 2000, pp. 2289-2293. doi:10.1039/b003221m

[4] J. Zhang, Z. Liu, B. Han, Z. Li, G. Yang, J. Li and J. Chen, "Preparation of Silica and $\mathrm{TiO}_{2}-\mathrm{SiO}_{2}$ Core-Shell Nanoparticles in Water-in-Oil Microemulsion Using Compressed $\mathrm{CO}_{2}$ as Reactant and Antisolvent," Journal of Supercritical Fluids, Vol. 36, No. 3, 2006, pp. 194-201. doi:10.1016/j.supflu.2005.06.002

[5] A. Palaniappan, J. Zhang, X. Su and F. E. H. Tay, "Preparation of Mesoporous Silica Films Using Sol-Gel Process and Argon Plasma Treatment," Chemical Physics Letters, Vol. 395, No. 1-3, 2004, pp. 70-74. doi:10.1016/j.cplett.2004.07.060

[6] C. Pijolat, J.P. Viricelle, G. Tournier and P. Montmeat, "Application of Membranes and Filtering Films for Gas Sensors Improvements," Thin Solid Films, Vol. 490, No. 1, 2005, pp. 7-16. doi:10.1016/j.tsf.2005.04.017

[7] M. H. Jo, J. K. Hong, H. H. Park, J. J. Kim, S. H. Hyun and S. Y. Choi, "Application of $\mathrm{SiO}_{2}$ Aerogel Film with Low Dielectric Constant to Intermetal Dielectrics," Thin Solid Films, Vol. 308-309, 1997, pp. 490-494. doi:10.1016/S0040-6090(97)00437-9

[8] B. J. O'sullivan, P. K. Hurley, C. Leveagle and J. H. Das, "Si-SiO ${ }_{2}$ Interface Properties Following Rapid Thermal Processing," Journal of Applied Physics, Vol. 89, No. 7, 2001, pp. 3811-3821. doi:10.1063/1.1343897

[9] M. Ritala, K. Kukli, A. Rahtu, P. Risnen, M. Leskel, T. Sajavaara and J. Keinonen, "Atomic Layer Deposition of Oxide Thin Films with Metal Alkoxides as Oxygen Sources," Science, Vol. 288, No. 5464, 2000, pp. 319-321. doi:10.1126/science.288.5464.319

[10] S. Tanaka, N. Nishiyama, Y. Oku, Y. Egashira and K. Ueyama, "Nano-Architectural Silica Thin Films with Two-Dimensionally Connected Cagelike Pores Synthesized from Vapor Phase," Journal of the American Chemical Society, Vol. 126, No. 15, 2004, pp. 4854-4858. doi:10.1021/ja039267z

[11] H. Yang, N. Coombs, I. Sokolov and G. A. Ozin, "FreeStanding and Oriented Mesoporous Silica Films Grown at the Air-Water Interface," Nature, Vol. 381, No. 6583, 1996, pp. 589-592. doi:10.1038/381589a0

[12] Q. Liu, J. Zhang, Q. Liu, Z. Zhu and J. Chen, "Sol-Gel Synthesis and Characterization of Silica Film with Two Opposite Structures: Nano-Porous and Protuberant," $M a-$ terials Chemistry and Physics, Vol. 114, No. 1, 2009, pp. 
309-312. doi:10.1016/j.matchemphys.2008.09.056

[13] Z. Qian, G. Hu, S. Zhang and M. Yang, "Preparation and Characterization of Montmorillonite-Silica Nanocomposites: A Sol-Gel Approach to Modifying Clay Surfaces,"” Physica B, Vol. 403, No. 18, 2008, pp. 3231-3238. doi:10.1016/j.physb.2008.04.008

[14] J. Sun, L. Qiao, S. Sun and G. Wang, "Photocatalytic Degradation of Orange $\mathrm{G}$ on Nitrogen-Doped $\mathrm{TiO}_{2}$ Catalysts under Visible Light and Sunlight Irradiation," Journal of Hazardous Materials, Vol. 155, No. 1-2, 2008, pp. 312-319. doi:10.1016/j.jhazmat.2007.11.062

[15] P. S. Singh, "High Surface Area Nanoporous Amorphous Silica Prepared by Dodecanol Assisted Silica Formate
Sol-Gel Approach," Journal of Colloid and Interface Science, Vol. 325, No. 1, 2008, pp. 207-214. doi:10.1016/j.jcis.2008.05.037

[16] K. M. S. Meera, R. M. Sankar, A. Murali, S. N. Jaisankar and A. B. Mandal, "Sol-Gel Network Silica/Modified Montmorillonite Clay Hybrid Nanocomposites Hydrophobic Surface Coatings," Colloids and Surfaces B: Biointerfaces, Vol. 90, 2012, pp. 204-210. doi:10.1016/i.colsurfb.2011.10.018

[17] S. K. Parida, S. Dash, S. Patel and B. K. Mishra, "Adsorption of Organic Molecules on Silica Surface," Advances in Colloid and Interface Science, Vol. 121, No. 1-3, 2006, pp. 77-110. doi:10.1016/j.cis.2006.05.028 